\title{
Human Gut Microbiota Associated with Obesity in Chinese Children and Adolescents
}

\author{
Ya-Ping Hou, ${ }^{1,2}$ Qing-Qing He, ${ }^{3}$ Hai-Mei Ouyang, ${ }^{4}$ Hai-Shan Peng, ${ }^{1,2}$ Qun Wang, ${ }^{3}$ Jie Li, ${ }^{3}$ \\ Xiao-Fei Lv, ${ }^{4}$ Yi-Nan Zheng, ${ }^{4}$ Shao-Chuan Li, ${ }^{3}$ Hai-Liang Liu, ${ }^{3}$ and Ai-Hua Yin ${ }^{1,2}$ \\ ${ }^{1}$ Medical Genetic Centre, Guangdong Women and Children Hospital, Guangzhou, Guangdong 510010, China \\ ${ }^{2}$ Maternal and Children Metabolic-Genetic Key Laboratory, Guangdong Women and Children Hospital, \\ Guangzhou, Guangdong 510010, China \\ ${ }^{3}$ CapitalBio Genomics Co., Ltd., Dongguan 532808, China \\ ${ }^{4}$ Department of Pediatrics, Guangdong Women and Children Hospital, Guangzhou, Guangdong 510010, China
}

Correspondence should be addressed to Hai-Liang Liu; hlliu@capitalgenomics.com and Ai-Hua Yin; yinaiwa@vip.126.com

Received 5 June 2017; Revised 4 September 2017; Accepted 27 September 2017; Published 29 October 2017

Academic Editor: Koichiro Wada

Copyright (C) 2017 Ya-Ping Hou et al. This is an open access article distributed under the Creative Commons Attribution License, which permits unrestricted use, distribution, and reproduction in any medium, provided the original work is properly cited.

\begin{abstract}
Objective. To investigate the gut microbiota differences of obese children compared with the control healthy cohort to result in further understanding of the mechanism of obesity development. Methods. We evaluated the 16S rRNA gene, the enterotypes, and quantity of the gut microbiota among obese children and the control cohort and learned the differences of the gut microbiota during the process of weight reduction in obese children. Results. In the present study, we learned that the gut microbiota composition was significantly different between obese children and the healthy cohort. Next we found that functional changes, including the phosphotransferase system, ATP-binding cassette transporters, flagellar assembly, and bacterial chemotaxis were overrepresented, while glycan biosynthesis and metabolism were underrepresented in case samples. Moreover, we learned that the amount of Bifidobacterium and Lactobacillus increased among the obese children during the process of weight reduction. Conclusion. Our results might enrich the research between gut microbiota and obesity and further provide a clinical basis for therapy for obesity. We recommend that Bifidobacterium and Lactobacillus might be used as indicators of healthy conditions among obese children, as well as a kind of prebiotic and probiotic supplement in the diet to be an auxiliary treatment for obesity.
\end{abstract}

\section{Introduction}

Obesity is a condition mainly caused by an alteration in energy intake, shifting towards positive energy balance, which can be influenced by genetic and environmental factors [1]. The prevalence of obesity is increasing at an epidemic rate globally, with more than one billion adults overweight and at least 300 million of them clinically obese [2]. Morbid obesity is known to be accompanied by serious health conditions, including hypertension, type 2 diabetes, cardiovascular diseases, stroke, and venous thromboembolism [3].

A large number of diverse microbial species reside in the distal gastrointestinal tract, and gut microbiota dysbiosis results in the imbalance of the composition and function of these intestinal microbes [4]. To date, many studies demonstrate that gut microbes play a great role in disease, ranging from localized gastroenterologic disorders to neurologic, respiratory, hepatic, and cardiovascular illness and metabolic related syndromes [5-8]. In particular, the fundamental role of gut microbiota in regulation and pathogenesis of metabolic disorders has attracted interest of more and more researchers in recent years. For the aspect of obesity, the research team of Jeffrey Gordon learned that differences of gut microbial communities were associated with obesity and that obesity would lead to reducing the diversity of microorganisms [9]. Subsequently, they confirmed the great role of gut microbes in obesity with an animal model in mice [10]. Dietary habits have strong influences on the selection of gut microbiota. Recently, proposed mechanisms about how the gut microbiota contributes to obesity, including the microbiota of obese subjects, had higher capacity to harvest energy from the diet providing substrates that can 
activate lipogenic pathways and, in addition, influence the activity of lipoprotein lipase interfering in the accumulation of triglycerides in the adipose tissue [11]. The gut microbiota has been proposed as another environmental factor involved in the onset of obesity. However, to which extent and through which mechanism the gut microbiota contributes to obesity development have not yet been elucidated.

In this present study, we recruited 87 obese children and adolescents as the case group and 56 healthy children and adolescents as the control group to analyze the community composition of gut microbiota between them and to further reveal potential microbial functional characteristics of obese children.

\section{Method and Materials}

2.1. Cohort in This Study. A total of 87 obese children (3-18 years) were enrolled in this study. In addition, 56 healthy children (3-18 years) were recruited as the healthy controls. Eleven of the obese children received an intervention in the hospital according to a dietary formula used in a previous study [12] and were resampled 30, 60, and 90 days after the intervention. None of the cohorts took antibiotics within the 3 months prior to sampling.

2.2. DNA Extraction and High-Throughput Sequencing. Community DNA was extracted from fecal samples using TIANamp Stool DNA Kit (TIANGEN, China), according to the manufacturer's instructions. We amplified the $\mathrm{V} 3$ region of the 16S rRNA gene by PCR with the following cycling conditions: a denaturing cycle of $98^{\circ} \mathrm{C}$ for $2 \mathrm{~min}$, followed by 35 cycles of $94^{\circ} \mathrm{C}$ for $30 \mathrm{~s}, 55^{\circ} \mathrm{C}$ for $30 \mathrm{~s}$, and $72^{\circ} \mathrm{C}$ for $30 \mathrm{~s}$. Amplified products were recycled with Agencourt AMPure $\mathrm{XP}$ kit and the PCR products were sequenced with the Ion PGM platform (Life, USA).

2.3. Data Processing. We filtered out the reads below the criteria of read quality not less than 25 , length between 100 and $500 \mathrm{bp}$, homopolymers less than 6 , and mismatches no more than 2. High-quality reads were clustered to operational taxonomic units (OTUs) (closed-reference) and annotated with taxonomy information from Greengene (ver.13_5) using QIIME pipeline [13]. Reads assigned to OTUs were rarefied with 20,000 per library (minimum reads among all samples) and then normalized by the 16S rRNA gene copy numbers using PICRUSt [14]. We generated a KO (Kyoto Encyclopedia of Genes and Genomes (KEGG) orthology) profile with PICRUSt and grouped KOs into metabolic pathway levels for subsequent statistical analysis.

2.4. Statistical Analysis. Principal coordinate analysis (PCoA) was performed with weighted-UniFrac distance matrix of all normalized OTUs through QIIME. The OTUs with occurring frequency among all time-scale samples over $40 \%$ were set as core OTUs. We clustered the core OTUs using the Ward clustering algorithm based on Spearman correlation coefficient distance matrix ( 1 - correlation coefficient) and determined the $P$ values for each node with the Permutational
MANOVA method (9999 permutations, $P<0.01$ ) using $\mathrm{R}$ package vegan. Genus-level markers were identified with Wilcoxon's rank sum test and effect sizes of LDA algorithm using LEfSe [15].

\section{Results}

In this study, the $16 \mathrm{~S}$ rRNA gene V3 hypervariable region was sequenced on the Ion PGM platform. We generated 8,580,178 raw reads with a median read length of 175 base pairs. After quality trimming and chimer filtering, 7,061,487 high-quality sequences remained and accounted for an average of 40,122 reads (range from 20,103 to 62,253) per sample. When we randomly selected 20,000 reads 10 times from all samples, the median average value of Good's coverage was nearly $98.65 \%$, which was close to the plateau (see Figure S1 in Supplementary Material available online at https://doi.org/10.1155/2017/ 7585989), indicating the sequencing depth was sufficient for characterizing the bacterial population in our fecal samples.

3.1. Gut Microbiota Dysbiosis in Obese Children. To determine how the gut microbial community shifts in obese children, we compared the overall structure of the fecal microbiota in the obese population with that of the healthy control population. Unexpectedly, obese children, not obese adults, did not exhibit a significant decrease in the fecal community diversity and richness, but the community structure of the fecal microbiota was quite different between the obese and healthy control group. Weighted-UniFrac distance was calculated to measure the similarity between microbial communities, which considered both the composition structure and the abundance of microbiota. Noticeably, the fecal microbiota from the obese group could be separated from those of the control group with principal coordinate analysis $(P$ value $<0.01$, Figure 1$)$.

3.2. Obese-Associated Gut Microbes in Children. The taxonomy of the fecal microbiota was assessed by a taxondependent analysis. Eighteen phyla were detected in our fecal samples, including 13 phyla observed in the obese cohort and 16 phyla observed in the normal control cohort (Figure S2). Firmicutes and Bacteroidetes were the predominant fecal microbiome in both cohorts. However, the relative abundance ratio of Firmicutes and Bacteroidetes (F/B) in the obese cohort was significantly higher than in the normal control cohort. The phyla of Verrucomicrobia, TM7, and Lentisphaerae had a median relative abundance $<0.1 \%$, which was significantly lower than those in the control group.

To identify the specific bacteria taxa associated with childhood obesity, we compared OTUs in case and control groups using LEfSe. A cladogram representative of the structure of the fecal microbiota was shown in Figure 2. The data indicated that intestinal dysbiosis was extensive in obese children. In the obese children, microbial assemblages were notably enriched in Firmicutes, especially Enterococcus and Blautia, as well as Sutterella and Klebsiella from Proteobacteria and Collinsella from Actinobacteria, while Bacteroides and Parabacteroides from Bacteroidetes, as well as Anaerotruncus 


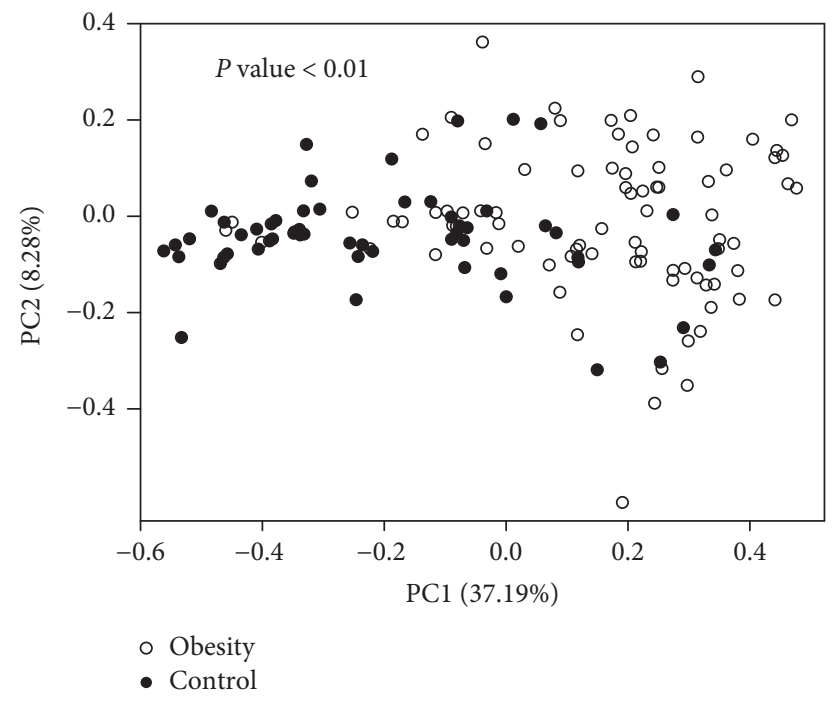

FIGURE 1: Composition comparison of fecal microbiota between the obese and control group. Plot of fecal microbiota based on the weighted UniFrac metric with principal coordinate analysis results.

and Coprobacillus from Firmicutes were enriched in healthy controls (data in Table S1).

3.3. Potential Microbial Functional Characteristics of Obese Children. To investigate the relationship between obese and gut microbiome functions, we predicted the potential metagenomes from the community profiles of normalized $16 \mathrm{~S}$ rRNA genes with PICRUSt [14]. The inferred gene families (KEGG Orthology groups) were annotated and combined with level 2 and level 3 pathways for statistic testing and visualization (Figure 3 and Table S2). Combined KEGG pathways and PICRUSt analysis results included categories associated with membrane transport and cell motility, which were overrepresented, while glycan biosynthesis and metabolism were underrepresented in case samples. Level 3 pathways in membrane transport consisted of the phosphotransferase system (PTS), ATP-binding cassette transporters (ABC transporters), and cell motility that contained flagellar assembly and bacterial chemotaxis.

3.4. Assessing the Functional Gut Microbes after Taking Dietary Therapy. Eleven of the obese children in our study received an intervention in the hospital according to a dietary formula used in a previous study and were resampled 30,60, and 90 days after the intervention. When analyzed, we divided the core OTUs into groups by Ward's hierarchical clustering based on Spearman's correlation distance. Each node of the dendrogram was tested using a permutational method and nine significant nodes (clusters) were retrieved. We found that the abundance of Node 10 displayed significant differences among samples of $0,30,60$, and 90 days during the process of weight reduction (Wilcoxon's rank sum test, $P$ $<0.01$ ) (Figure 4). Node 10 mostly comprised members of the genus Bifidobacterium and Lactobacillus (Table S3).

\section{Discussion}

Obesity is a metabolic disease that is often accompanied by dyslipidemia, hypertension, and impaired glucose homeostasis, and it has been reported to be associated with intestinal dysbiosis $[1,16]$. In knockout and diet-induced obese mice, obesity was associated with changes in the composition and metabolic function of the gut microbiota [17]. Dysbiosis is a form of altered gut metagenome and collected microbial activities, and, in combination with classic genetic and environmental factors, it may promote the development of metabolic disorders [18]. To deeply understand obesity and the metabolic mechanism related to gut microbiota would make an improvement in the treatment of obesity.

4.1. Gut Microbiota Dysbiosis in Obese Children. Here, we recruited 87 obese children and adolescents as a case group and 56 healthy children and adolescents as a control group to analyze the gut microbiota differences between them. Based on our results, obese children here did not exhibit a significant decrease in the fecal community diversity and richness, while being different from the results based on obese adults. However, the composition of the gut microbiota showed significant differences between obese children and healthy controls. First, our results indicated that the phyla of Firmicutes and Bacteroidetes were the predominant fecal microbiome in both cohorts. However, the relative abundance ratio of Firmicutes and Bacteroidetes (F/B) in the obese cohort was significantly higher than that in the healthy controls (Figure S2), which was consistent with the study in obese adults [19]. Firmicutes is associated with genes involved in carbohydrate catabolism and is rich in obese individuals [20], while Bacteroidetes is linked with diminished body mass [21]. Second, we also found some specific bacteria exhibited significant intergroup variations in their abundance, with changes in Enterococcus, Blautia, Sutterella, Klebsiella, and Collinsella, which were enriched in obese children, while Bacteroides, Parabacteroides, Anaerotruncus, and Coprobacillus were enriched in healthy controls (Table S1). Turnbaugh et al. also found that after mice were fed a low-fat, plant polysaccharide-rich diet and then switched to a Western diet, the microbiota composition shifted to an overgrowth of Firmicutes, including Clostridium innocuum, Eubacterium dolichum, Catenibacterium mitsuokai, and Enterococcus spp., and to a significant reduction in several Bacteroides spp. [22]. Case-specific markers in our study were found to be associated with a high-fat Western diet from previous research and might be a contributing factor to obesity. In addition, bacteria belonging to the genera Collinsella have been reported to be positively correlated with insulin [23] and that it was enriched in T2D patients [24]; therefore, enrichment of Collinsella here might imply the possibility of insulin resistance in obese children. In addition, Blautia producta was suggested to be related to a high-fat diet causing obesity in a mouse model [25].

4.2. Obese Related Functional Change. Intestinal dysbiosis in obese children would lead to functional change. With the KEGG pathway results (Figure 3 and Table S2), we 


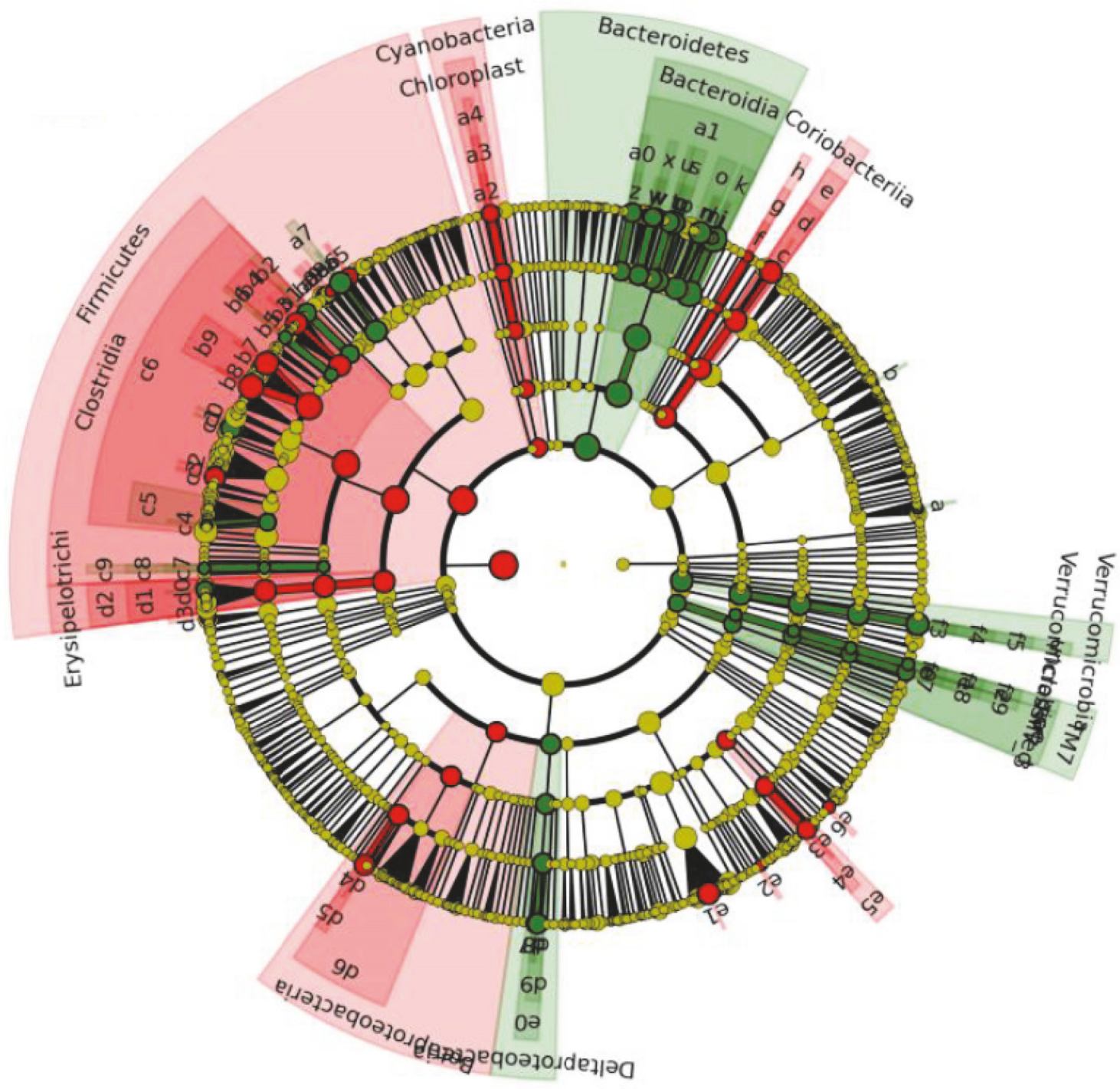

Case

Control

a: Mobiluncus

b: Kocuria

c: Collinsella

d: Coriobacteriaceae

e: Coriobacteriales

f: unclassified

g: Nitriliruptoraceae

h: Nitriliruptorales

i: Bacteroides

j: unclassified

$\mathrm{k}$ : Bacteroidaceae

1: Dysgonomonas

$\mathrm{m}$ : Paludibacter

n: Parabacteroides

o: Porphyromonadaceae

p: AF12

q: Rikenella

r: unclassified s: Rikenellaceae

t: unclassified

u: S24_7

v: Butyricimonas

w: Odoribacter

$\mathrm{x}$ : Odoribacteraceae

$y:$ CF231

z: unclassified

a0: unclassified

a1: Bacteroidales

a2: unclassified

a3: unclassified

a4: Streptophyta

a5: Enterococcus

a6: Lactobacillus

a7: Lactobacillaceae

a8: Weissella

a9: Lactococcus

b0: unclassified

b1: unclassified

Figure 2: Community population differences between the obese and control group. Enrichment microbes from obese samples are marked red, while the control samples are marked green. 


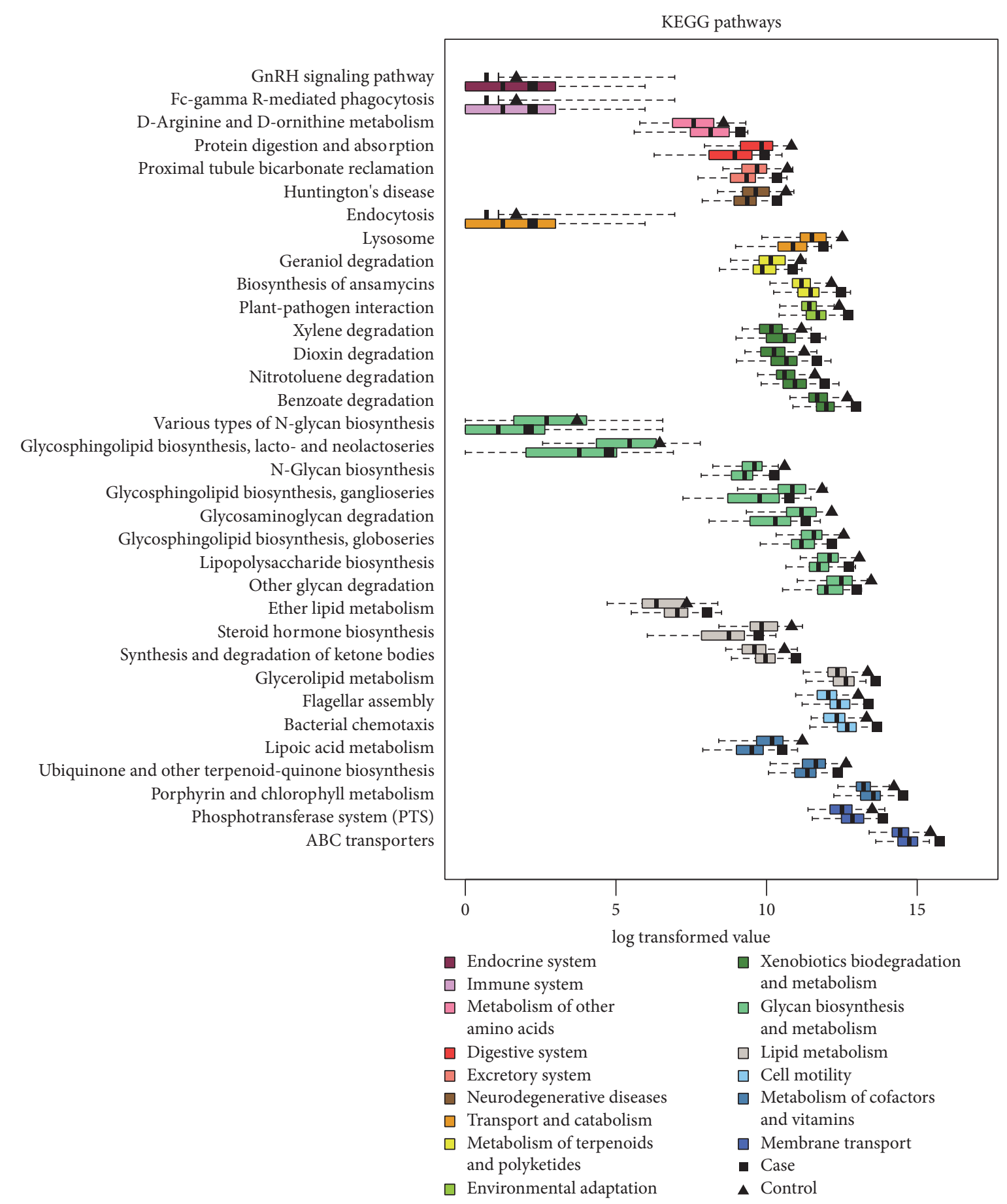

FIGURE 3: Functional changes derived from the obesity-associated gut microbiota. All significantly different level 3 pathways are illustrated and colored according to level 2 pathway categories. Ordinate indicated metabolic pathways; different colors represent the classification of different metabolic pathways. Each pathway has two box figures, which represent the expression of obese children and control samples in this metabolic pathway. Significant changes in the relative abundance of estimated genes summarized by KEGG pathway annotations between obese children and controls are shown with $P$ values $<0.05$.

knew that the activities of membrane transport and cell motility were overrepresented, while that of glycan biosynthesis and metabolism were underrepresented in case samples. Among these signaling pathways, phosphotransferase systems from the related pathway of membrane transport were more active in obese children. Phosphotransferase systems (PTS) are a class of transport systems involved in the uptake and phosphorylation of a variety of carbohydrates. The PTS also plays a role in regulating microbial gene expression through catabolite repression, allowing the cell to preferentially import simple sugars over other carbohydrates $[26,27]$. Moreover, functional analysis revealed that the 
obese human gut microbiome was enriched with phosphotransferase systems involved in microbial processing of carbohydrates primarily present in Actinobacteria and Firmicutes $[9,28]$. In addition, ATP-binding cassette transporters (ABC transporters) from the related pathway of membrane transport were also active. $\mathrm{ABC}$ transporters are members of a transport system super family in all extant phyla from prokaryotes to humans [29]. ABC uptake transporters could uptake a large variety of nutrients, biosynthetic precursors, trace metals, and vitamins, while exporters transport sterols, lipids, drugs, and a large variety of primary and secondary metabolites. Some of these exporters in humans are involved in tumor resistance, cystic fibrosis, and a range of other inherited human diseases [30]. The $\mathrm{ABC}$ transporter related diseases include Alzheimer's disease and drug resistance of malignant tumors [31,32]. However, this was the first time to be mentioned that $\mathrm{ABC}$ transporters have a relationship with obesity, here. More evidence and further mechanisms need to be provided next.

Second, cell motility, which involved flagellar assembly, and bacterial chemotaxis were second active in obese children. In 2009, Hildebrandt et al. found that a collection of gene groups linked to bacterial motility increased on a high-fat diet, including "flagellar motility" and "motility and chemotaxis" [33].

Last, biosynthesis of various types of $\mathrm{N}$-glycans, glycosphingolipids, lipopolysaccharide (LPS), and degradation of glycosaminoglycans (GAGs) and other glycans were found to be lower here in obese children compared with the healthy controls. N-glycan biosynthesis disorders could cause metabolic diseases, such as classical galactosemia (OMIM \#230400) [34]. Glycan biosynthesis and metabolism disorders could lead to a wide range of carbohydrate imbalance in the human body, hence, being known to play a great role in change in weight reduction [35]. LPS is a cell wall component of Gramnegative bacteria, which lyses into plasma when the bacteria die. Here, the phyla of Verrucomicrobia and Lentisphaerae, belonging to Gram-negative bacteria, were significantly lower in obese group than those in the control group, which may lead to lower level of LPS in the obese children. Cani et al. found that LPS, which acts on the innate immune system response in adipose tissue in obesity, is the triggering factor of the early development of inflammation and metabolic diseases [36]. Importantly, Verrucomicrobia acts as a polysaccharide degrader and is proposed as a hallmark of a healthy gut due to its anti-inflammatory and immunostimulant properties and its ability to improve gut barrier function, insulin sensitivity, and endotoxinemia [37]. Researchers studied that the inflammation is not all bad for obesity, and it would play a positive role by stimulating energy expenditure and facilitating adipose tissue remodeling $[38,39]$. Obesity is associated with low-grade inflammation in adipose tissue, which has been implicated in the development of metabolic syndrome and insulin resistance. Therefore, low-LPS could disrupt the energy consumption and adipose tissue remodeling.

4.3. Gut Microbiota Changes under the Process of Weight Reduction. Upon analyzing the gut microbiota changes after $0,30,60$, and 90 days during the process of weight reduction

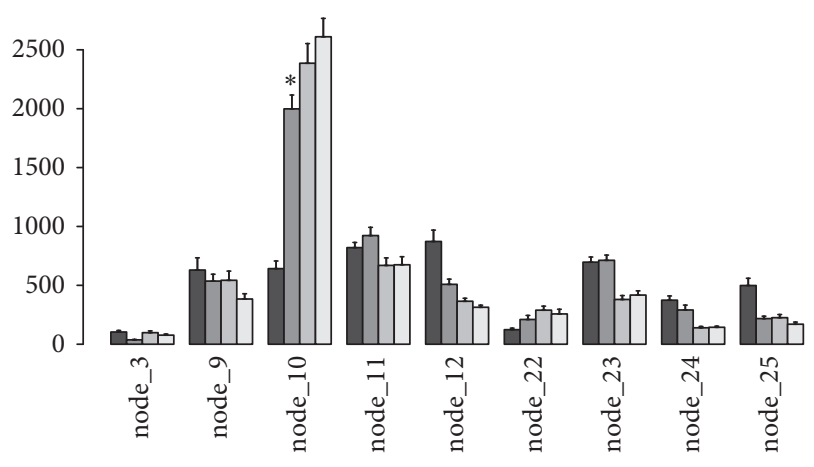

FIGURE 4: Abundance results of significant clusters of core OTUs for samples during 0, 30, 60, and 90 days of the dietary intervention. The asterisk indicates the statistical significance of $P$ value $<0.01$. OTUs species information in node_10 is listed in Table S3. ${ }^{*}$ indicates the statistical significance of $P$ value $<0.01$ compared with the dark grey sample (as control) in node_10.

in eleven obese children, who in our study received an intervention in a hospital according to a dietary formula used in a previous study, we found that the abundance of Node 10 displayed significant differences $(P$ value $<0.01)$ (Figure 4), which mostly contained members of the genus Bifidobacterium and Lactobacillus (Table S3). As reported before, dietary oligosaccharides derived from plant and milk stimulate the growth of Bifidobacterium, which is linked to normal weight gain and were found in low concentrations in obese individuals [40]. Bifidobacterium could reduce obesity-associated inflammation by restoring the lymphocyte-macrophage balance and reduce the abundance of Firmicutes, in which these effects were accompanied by reductions in body weight gain and in serum cholesterol, triglycerides, glucose, and insulin levels and improved oral glucose tolerance and insulin sensitivity in obese mice [41]. In addition, Lactobacillus played a dual function in preventing high-fat-diet-induced obesity, including direct reduction of cholesterol and upregulation of PPARalpha in adipose tissue $[42,43]$. Therefore, promoting changes in the gut microbiota might play an important role in ensuring the efficacy and success of obesity treatments. To our knowledge, Bifidobacterium and Lactobacillus might be used as indicators of healthy conditions among obese children and adolescents, as well as a kind of prebiotic and probiotic supplement in the diet to be an auxiliary treatment in obesity.

\section{Conclusion}

In summary, from the present study, we learned that the gut microbiota composition was significantly different between obese children and the healthy cohort, which included bacteria categories of Enterococcus, Blautia, Sutterella, Klebsiella, and Collinsella, which were enriched in obese children, while Bacteroides, Parabacteroides, Anaerotruncus, and Coprobacillus were enriched in healthy controls. Then, we knew that functional changes, including PTS and $\mathrm{ABC}$ transporter related membrane transport and flagellar assembly and bacterial chemotaxis related cell motility, were overrepresented, 
while glycan biosynthesis and metabolism were underrepresented in case samples. Moreover, we learned that the amount of Bifidobacterium and Lactobacillus increased among the obese children during the process of weight reduction. Our results might enrich the research between gut microbiota and obesity, further providing a clinical basis for therapy for obesity. Finally, we recommend that Bifidobacterium and Lactobacillus might be used as indicators of healthy conditions among obese children and adolescents, as well as a kind of prebiotic and probiotic supplement in the diet to be an auxiliary treatment for obesity.

\section{Ethical Approval}

This study was approved by the Ethics Committee of Guangdong Women and Children Hospital, Guangzhou, Guangdong 510010, China.

\section{Disclosure}

Ya-Ping Hou and Qing-Qing He are co-first authors.

\section{Conflicts of Interest}

The authors declare no conflicts of interest.

\section{Authors' Contributions}

All authors have materially participated in the research and/or the article preparation. Ai-Hua Yin, Hai-Liang Liu, and Ya-Ping Hou conceived and designed the experiments; Hai-Mei Ouyang, Hai-Shan Peng, and Xiao-Fei Lv performed the experiments; Qun Wang, Jie Li, Qing-Qing He, and ShaoChuan Li analyzed the data; Ya-Ping Hou and Yi-Nan Zheng contributed reagents/materials/analysis tools; Qing-Qing He and Hai-Liang Liu wrote and submitted the paper. All authors have approved the final article. Ya-Ping Hou and Qing-Qing He contributed to this work equally.

\section{Acknowledgments}

The authors thank the Guangdong Provincial Science and Technology Plan Project (Grant no. 2014A020213019).

\section{References}

[1] A. A. Lopez-Cepero and C. Palacios, "Association of the intestinal microbiota and obesity," Puerto Rico Health Sciences Journal, vol. 34, pp. 60-64, 2015.

[2] K. Ghoorah, P. Campbell, A. Kent, A. Maznyczka, and V. Kunadian, "Obesity and cardiovascular outcomes: A review," European Heart Journal: Acute Cardiovascular Care, vol. 5, no. 1, pp. 77-85, 2016.

[3] G. Yang, C. D. Staercke, and W. C. Hooper, "The effects of obesity on venous thromboembolism: a review," Open Journal of Preventive Medicine, vol. 02, no. 04, pp. 499-509, 2012.

[4] S. V. Lynch and O. Pedersen, "The human intestinal microbiome in health and disease," The New England Journal of Medicine, vol. 375, no. 24, pp. 2369-2379, 2016.
[5] K. Guyton and J. C. Alverdy, "The gut microbiota and gastrointestinal surgery," Nature Reviews Gastroenterology \& Hepatology, vol. 14, no. 1, pp. 43-54, 2017.

[6] C. Leung, L. Rivera, J. B. Furness, and P. W. Angus, "The role of the gut microbiota in NAFLD," Nature Reviews Gastroenterology \& Hepatology, vol. 13, no. 7, pp. 412-425, 2016.

[7] K. V. Sandhu, E. Sherwin, H. Schellekens, C. Stanton, T. G. Dinan, and J. F. Cryan, "Feeding the microbiota-gut-brain axis: diet, microbiome, and neuropsychiatry," Translational Research, vol. 179, pp. 223-244, 2017.

[8] W. H. W. Tang and S. L. Hazen, "Dietary metabolism, gut microbiota and acute heart failure," Heart, vol. 102, no. 11, pp. 813-814, 2016.

[9] P. J. Turnbaugh, M. Hamady, T. Yatsunenko et al., "A core gut microbiome in obese and lean twins," Nature, vol. 457, no. 7228, pp. 480-484, 2009.

[10] V. K. Ridaura, J. J. Faith, and F. E. Rey, "Gut microbiota from twins discordant for obesity modulate metabolism in mice," Science, vol. 341, no. 6150, Article ID 1241214, 2013.

[11] A. P. Boroni Moreira, T. Fiche Salles Teixeira, M. do C Gouveia Peluzio, and R. de Cássia Gonçalves Alfenas, "Gut microbiota and the development of obesity," Nutrición Hospitalaria, vol. 27, no. 5, pp. 1408-1414, 2012.

[12] C. Zhang, A. Yin, H. Li et al., "Dietary modulation of gut microbiota contributes to alleviation of both genetic and simple obesity in children," EBioMedicine, vol. 2, no. 8, pp. 968-984, 2015.

[13] J. G. Caporaso, J. Kuczynski, J. Stombaugh et al., "QIIME allows analysis of high-throughput community sequencing data," Nature Methods, vol. 7, no. 5, pp. 335-336, 2010.

[14] M. G. I. Langille, J. Zaneveld, J. G. Caporaso et al., "Predictive functional profiling of microbial communities using $16 \mathrm{~S}$ rRNA marker gene sequences," Nature Biotechnology, vol. 31, no. 9, pp. 814-821, 2013.

[15] N. Segata, J. Izard, L. Waldron et al., "Metagenomic biomarker discovery and explanation," Genome Biology, vol. 12, no. 6, article R60, 2011.

[16] E. E. Patterson, P. M. Ryan, J. F. Cryan et al., "Gut microbiota, obesity and diabetes," Postgraduate Medical Journal, vol. 92, no. 1087, pp. 286-300, 2016.

[17] H. Tilg, A. R. Moschen, and A. Kaser, "Obesity and the microbiota," Gastroenterology, vol. 136, no. 5, pp. 1476-1483, 2009.

[18] K. Harris, A. Kassis, G. Major, and C. J. Chou, "Is the gut microbiota a new factor contributing to obesity and its metabolic disorders?" Journal of Obesity, vol. 2012, Article ID 879151, 14 pages, 2012.

[19] A. Santacruz, M. C. Collado, L. García-Valdés et al., "Gut microbiota composition is associated with body weight, weight gain and biochemical parameters in pregnant women," British Journal of Nutrition, vol. 104, no. 1, pp. 83-92, 2010.

[20] P. J. Turnbaugh and J. I. Gordon, "The core gut microbiome, energy balance and obesity," The Journal of Physiology, vol. 587, no. 17, pp. 4153-4158, 2009.

[21] C. de Filippo, D. Cavalieri, M. di Paola et al., "Impact of diet in shaping gut microbiota revealed by a comparative study in children from Europe and rural Africa," Proceedings of the National Acadamy of Sciences of the United States of America, vol. 107, no. 33, pp. 14691-14696, 2010.

[22] P. J. Turnbaugh, V. K. Ridaura, J. J. Faith, F. E. Rey, R. Knight, and J. I. Gordon, "The effect of diet on the human gut microbiome: a 
metagenomic analysis in humanized gnotobiotic mice," Science Translational Medicine, vol. 1, no. 6, p. 6ral4, 2009.

[23] L. F. Gomez-Arango, H. L. Barrett, H. D. McIntyre et al., "Connections between the gut microbiome and metabolic hormones in early pregnancy in overweight and obese women," Diabetes, vol. 65, no. 8, pp. 2214-2223, 2016.

[24] X. Zhang, D. Shen, Z. Fang et al., "Human gut microbiota changes reveal the progression of glucose intolerance," PLOS ONE, vol. 8, no. 8, Article ID e71108, 2013.

[25] N. Becker, J. Kunath, G. Loh, and M. Blaut, "Human intestinal microbiota: characterization of a simplified and stable gnotobiotic rat model," Gut Microbes, vol. 2, no. 1, pp. 25-33, 2011.

[26] J. Deutscher, C. Francke, and P. W. Postma, "How phosphotransferase system-related protein phosphorylation regulates carbohydrate metabolism in bacteria," Microbiology and Molecular Biology Reviews, vol. 70, no. 4, pp. 939-1031, 2006.

[27] P. J. Turnbaugh, F. Bäckhed, L. Fulton, and J. I. Gordon, "Dietinduced obesity is linked to marked but reversible alterations in the mouse distal gut microbiome," Cell Host \& Microbe, vol. 3, no. 4, pp. 213-223, 2008.

[28] J. Graessler, Y. Qin, H. Zhong et al., "Metagenomic sequencing of the human gut microbiome before and after bariatric surgery in obese patients with type 2 diabetes: correlation with inflammatory and metabolic parameters," The Pharmacogenomics Journal, vol. 13, no. 6, pp. 514-522, 2013.

[29] O. Polgar and S. E. Bates, "ABC transporters in the balance: Is there a role in multidrug resistance?" Biochemical Society Transactions, vol. 33, no. 1, pp. 241-245, 2005.

[30] K. Beis, "Structural basis for the mechanism of ABC transporters," Biochemical Society Transactions, vol. 43, pp. 889-893, 2015.

[31] M. Dean, "ABC transporters, drug resistance, and cancer stem cells," Journal of Mammary Gland Biology and Neoplasia, vol. 14, no. 1, pp. 3-9, 2009.

[32] A. Wolf, B. Bauer, and A. M. Hartz, "ABC transporters and the alzheimer's disease enigma," Frontiers in Psychiatry, vol. 3, no. 54, 2012.

[33] M. A. Hildebrandt, C. Hoffmann, S. A. Sherrill-Mix et al., "High-fat diet determines the composition of the murine gut microbiome independently of obesity," Gastroenterology, vol. 137, no. 5, pp. 1716.e2-1724.e2, 2009.

[34] A. Maratha, H. Stockmann, K. P. Coss et al., "Classical galactosaemia: novel insights in IgG N-glycosylation and N-glycan biosynthesis," European Journal of Human Genetics, vol. 24, no. 7, pp. 976-984, 2016.

[35] A. Woting and M. Blaut, "The intestinal microbiota in metabolic disease," Nutrients, vol. 8, no. 4, article 202, 2016.

[36] P. D. Cani, J. Amar, M. A. Iglesias et al., "Metabolic endotoxemia initiates obesity and insulin resistance," Diabetes, vol. 56, no. 7, pp. 1761-1772, 2007.

[37] S. Fujio-Vejar, Y. Vasquez, P. Morales et al., "The gut microbiota of healthy chilean subjects reveals a high abundance of the phylum verrucomicrobia," Frontiers in Microbiology, vol. 8, 2017.

[38] G. S. Hotamisligil, "Inflammation and metabolic disorders," Nature, vol. 444, no. 7121, pp. 860-867, 2006.

[39] J. Ye and O. P. McGuinness, "Inflammation during obesity is not all bad: evidence from animal and human studies," American Journal of Physiology-Renal Physiology, vol. 304, no. 5, pp. E466E477, 2013.

[40] R. G. LoCascio, M. R. Ninonuevo, S. L. Freeman et al., "Glycoprofiling of bifidobacterial consumption of human milk oligosaccharides demonstrates strain specific, preferential consumption of small chain glycans secreted in early human lactation," Journal of Agricultural and Food Chemistry, vol. 55, no. 22, pp. 8914-8919, 2007.

[41] A. Moya-Pérez, A. Neef, and Y. Sanz, "Bifidobacterium pseudocatenulatum CECT 7765 reduces obesity-associated inflammation by restoring the lymphocyte-macrophage balance and gut microbiota structure in high-fat diet-fed mice," PLOS ONE, vol. 10, no. 7, Article ID e0126976, 2015.

[42] D. H. Kim, D. Jeong, I. B. Kang, H. Kim, K. Y. Song, and K. H. Seo, "Dual function of Lactobacillus kefiri DH5 in preventing high-fat-diet-induced obesity: direct reduction of cholesterol and upregulation of PPARalpha in adipose tissue," Molecular Nutrition \& Food Research, p. 1700252, 2017.

[43] S. Park, Y. Ji, H.-Y. Jung et al., "Lactobacillus plantarum HAC01 regulates gut microbiota and adipose tissue accumulation in a diet-induced obesity murine model," Applied Microbiology and Biotechnology, vol. 101, no. 4, pp. 1605-1614, 2017. 

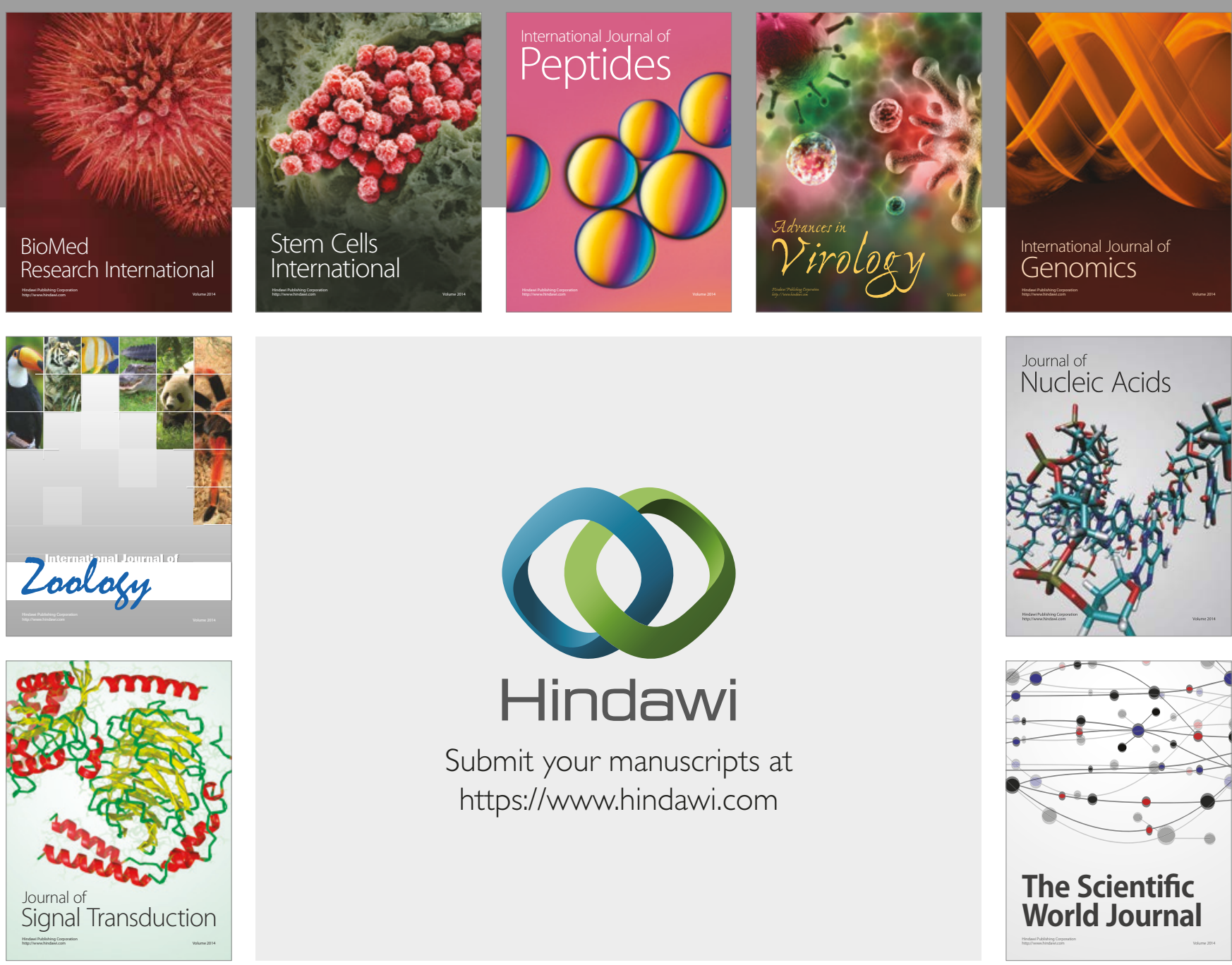

Submit your manuscripts at

https://www.hindawi.com
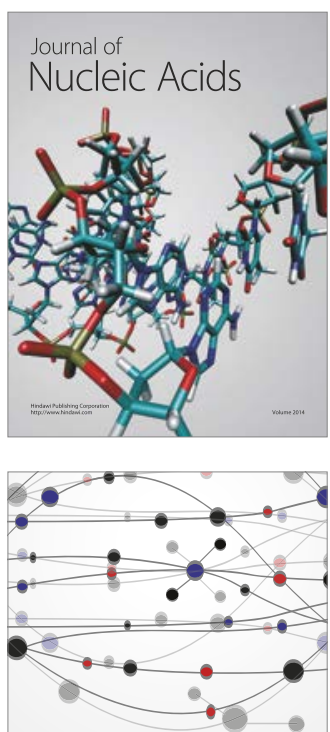

The Scientific World Journal

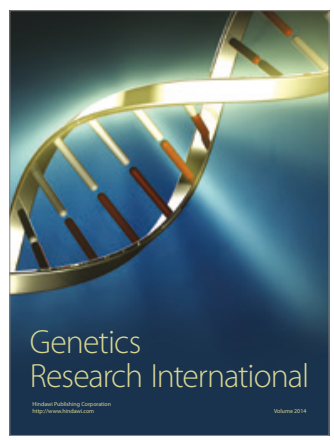

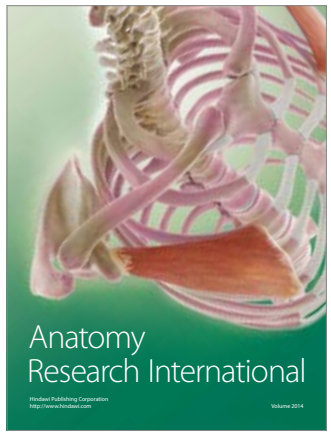

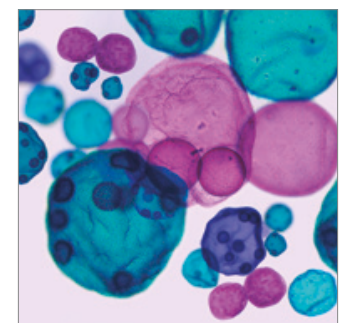

International Journal of Microbiology
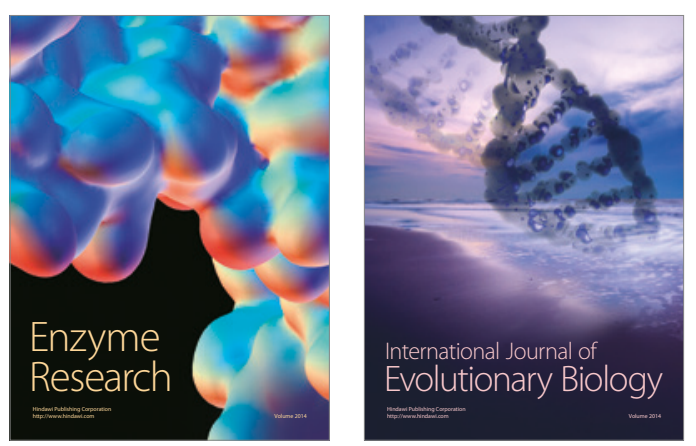
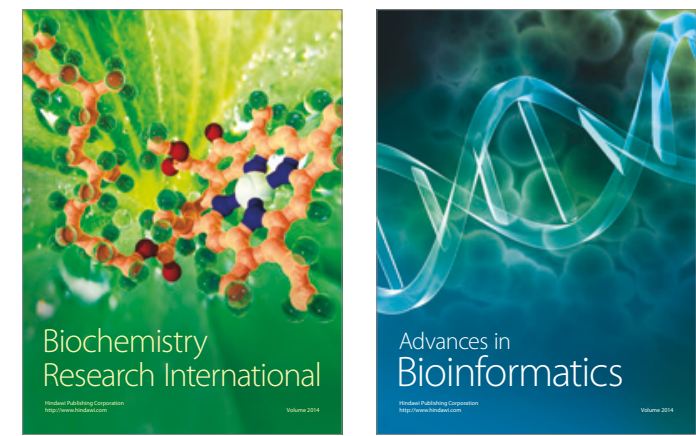

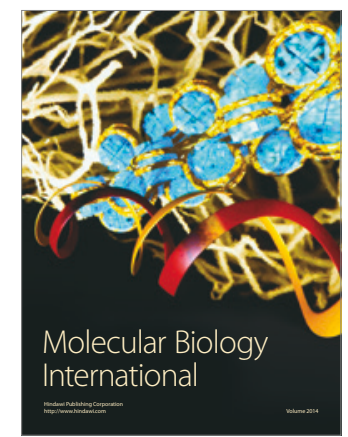

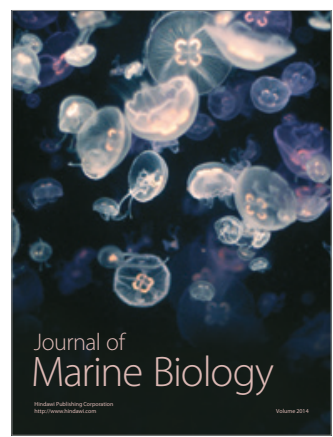

